\title{
Instable Trivial Solution of Autonomous Differential Systems with Quadratic Right-Hand Sides in a Cone
}

\author{
D. Ya. Khusainov, ${ }^{1} \mathrm{~J}$. Diblík, ${ }^{2,3}{ }^{3}$ Z. Svoboda, ${ }^{2}$ and Z. Šmarda $^{2}$ \\ ${ }^{1}$ Department of Complex System Modeling, Faculty of Cybernetics, Taras Shevchenko \\ National University of Kyiv, Vladimirskaya Str. 64, 01033 Kyiv, Ukraine \\ 2 Department of Mathematics, Faculty of Electrical Engineering and Communication, Technická 8, \\ Brno University of Technology, 61600 Brno, Czech Republic \\ ${ }^{3}$ Department of Mathematics and Descriptive Geometry, Faculty of Civil Engineering, Veveři 331/95, \\ Brno University of Technology, 60200 Brno, Czech Republic
}

Correspondence should be addressed to D. Ya. Khusainov, khusainov@stab.univ.kiev.ua

Received 5 October 2010; Accepted 2 November 2010

Academic Editor: Miroslava Růžičková

Copyright (c) 2011 D. Ya. Khusainov et al. This is an open access article distributed under the Creative Commons Attribution License, which permits unrestricted use, distribution, and reproduction in any medium, provided the original work is properly cited.

The present investigation deals with global instability of a general $n$-dimensional system of ordinary differential equations with quadratic right-hand sides. The global instability of the zero solution in a given cone is proved by Chetaev's method, assuming that the matrix of linear terms has a simple positive eigenvalue and the remaining eigenvalues have negative real parts. The sufficient conditions for global instability obtained are formulated by inequalities involving norms and eigenvalues of auxiliary matrices. In the proof, a result is used on the positivity of a general third-degree polynomial in two variables to estimate the sign of the full derivative of an appropriate function in a cone.

\section{Introduction}

Recently, there has been a rapidly growing interest in investigating the instability conditions of differential systems. The number of papers dealing with instability problems is rather low compared with the huge quantity of papers in which the stability of the motion of differential systems is investigated. The first results on the instability of zero solution of differential systems were obtained in a general form by Lyapunov [1] and Chetaev [2].

Further investigation on the instability of solutions of systems was carried out to weaken the conditions of the Lyapunov and Chetaev theorems for special-form systems. Some results are presented, for example, in [3-10], but instability problems are analysed only locally. For example, in [7], a linear system of ordinary differential equations in the matrix form is considered, and conditions such that the corresponding forms (of the second and the 
third power) have fixed sign in some cone of the space $\mathbb{R}^{n}$ are derived. To investigate this property another problem inverse to the known Lyapunov problem for the construction of Lyapunov functions is solved.

In the present paper, instability solutions of systems with quadratic right-hand sides is investigated in a cone dealing with a general $n$-dimensional system with quadratic righthand sides. We assume that the matrix of linear terms has a simple positive eigenvalue and the remaining eigenvalues have negative real parts.

Unlike the previous investigations, we prove the global instability of the zero solution in a given cone and the conditions for global instability are formulated by inequalities involving norms and eigenvalues of auxiliary matrices. The main tool is the method of Chetaev and application of a suitable Chetaev-type function. A novelty in the proof of the main result (Theorem 3.1) is the utilization of a general third-order polynomial inequality of two variables to estimate the sign of the full derivative of an appropriate function along the trajectories of a given system in a cone.

In the sequel, the norms used for vectors and matrices are defined as

$$
\|x\|=\left(\sum_{i=1}^{n} x_{i}^{2}\right)^{1 / 2}
$$

for a vector $x=\left(x_{1}, \ldots, x_{n}\right)^{T}$ and

$$
\|\mathscr{F}\|=\left(\lambda_{\max }\left(\mathcal{F}^{T} \mathcal{F}\right)\right)^{1 / 2}
$$

for any $m \times n$ matrix $\mathcal{F}$. Here and throughout the paper, $\lambda_{\max }(\cdot)$ (or $\left.\lambda_{\min }(\cdot)\right)$ is the maximal (or minimal) eigenvalue of the corresponding symmetric and positive-semidefinite matrix $\mathcal{F}^{T} \mathcal{F}$ (see, e.g., [11]).

In this paper, we consider the instability of the trivial solution of a nonlinear autonomous differential system with quadratic right-hand sides

$$
\dot{x}_{i}=\sum_{s=1}^{n} a_{i s} x_{s}+\sum_{s, q=1}^{n} b_{s q}^{i} x_{s} x_{q}, \quad i=1, \ldots, n,
$$

where coefficients $a_{i s}$ and $b_{s q}^{i}$ are constants. Without loss of generality, throughout this paper we assume

$$
b_{s q}^{i}=b_{q s}^{i} .
$$

As emphasized, for example, in [2,10-12], system (1.3) can be written in a general vectormatrix form

$$
\dot{x}=A x+X^{T} B x,
$$


where $A$ is an $n \times n$ constant square matrix, matrix $X^{T}$ is an $n \times n^{2}$ rectangular matrix

$$
X^{T}=\left\{X_{1}^{T}, X_{2}^{T}, \ldots, X_{n}^{T}\right\},
$$

where the entries of the $n \times n$ square matrices $X_{i}, i=1, \ldots, n$ are equal to zero except the $i$ th row with entries $x^{T}=\left(x_{1}, x_{2}, \ldots, x_{n}\right)$, that is,

$$
X_{i}^{T}=\left(\begin{array}{cccc}
0 & 0 & \cdots & 0 \\
\cdots & \cdots & \cdots & \cdots \\
0 & 0 & \cdots & 0 \\
x_{1} & x_{2} & \cdots & x_{n} \\
0 & 0 & \cdots & 0 \\
\cdots & \cdots & \cdots & \cdots \\
0 & 0 & \cdots & 0
\end{array}\right)
$$

and $B$ is a rectangular $n^{2} \times n$ matrix such that

$$
B^{T}=\left\{B_{1}, B_{2}, \ldots, B_{n}\right\},
$$

where matrices $B_{i}=\left\{b_{s q}^{i}\right\}, i, s, q=1, \ldots, n$, that is, matrices

$$
B_{i}=\left(\begin{array}{cccc}
b_{11}^{i} & b_{12}^{i} & \cdots & b_{1 n}^{i} \\
b_{21}^{i} & b_{22}^{i} & \cdots & b_{2 n}^{i} \\
\cdots & \cdots & \cdots & \cdots \\
b_{n 1}^{i} & b_{n 2}^{i} & \cdots & b_{n n}^{i}
\end{array}\right)
$$

are $n \times n$ constant and symmetric. Representation (1.5) permits an investigation of differential systems with quadratic right-hand sides by methods of matrix analysis. Such approach was previously used, for example, in [13].

If matrix $A$ admits one simple positive eigenvalue, the system (1.5) can be transformed, using a suitable linear transformation of the dependent variables, to the same form (1.5) but with the matrix $A$ having the form

$$
A=\left(\begin{array}{ll}
A_{0} & \theta \\
\theta^{T} & \lambda
\end{array}\right),
$$

where $A_{0}$ is an $(n-1) \times(n-1)$ constant matrix, $\theta=(0,0, \ldots, 0)^{T}$ is the $(n-1)$-dimensional zero vector and $\lambda>0$. With regard to this fact, we do not introduce new notations for the coefficients $b_{s q}^{i}, i, s, q=1,2, \ldots, n$ in (1.5), assuming throughout the paper that $A$ in (1.5) has the form (1.10), preserving the old notations $a_{i j}$ for entries of matrix $A_{0}$. This means that we 
assume that $A=\left\{a_{i s}\right\}, i, s=1,2, \ldots, n$ with $a_{n s}=a_{s n}=0$ for $s=1,2, \ldots, n-1$ and $a_{n n}=\lambda$, and $A_{0}=\left\{a_{i s}\right\}, i, s=1,2, \ldots, n-1$.

We will give criteria of the instability of a trivial solution of the system (1.5) if the matrix $A$ of linear terms is defined by (1.10).

\section{Preliminaries}

In this part we collect the necessary material-the definition of a cone, auxiliary Chetaev-type results on instability in a cone and, finally, a third degree polynomial inequality, which will be used to estimate the sign of the full derivative of a Chetaev-type function along the trajectories of system (1.5).

\subsection{Instability of the Zero Solution of Systems of Differential Equations in a Cone}

We consider an autonomous system of differential equations

$$
\dot{x}=f(x),
$$

where $f: \mathbb{R}^{n} \rightarrow \mathbb{R}^{n}$ satisfies a local Lipschitz condition and $f(0)=0$, that is, (2.1) admits the trivial solution. We will consider solutions of (2.1) determined by points $(x, t)=\left(x_{0}, 0\right)$ where $x_{0} \in \mathbb{R}^{n}$. The symbol $x\left(x_{0}, t\right)$ denotes the solution $x=x(t)$ of (2.1), satisfying initial condition $x(0)=x_{0}$.

Definition 2.1. The zero solution $x \equiv 0$ of (2.1) is called unstable if there exists $\varepsilon>0$ such that, for arbitrary $\delta>0$, there exists an $x_{0} \in \mathbb{R}^{n}$ with $\left\|x_{0}\right\|<\delta$ and $T \geq 0$ such that $\left\|x\left(x_{0}, T\right)\right\| \geq \varepsilon$.

Definition 2.2. A set $K \subset R^{n}$ is called a cone if $\alpha x \in K$ for arbitrary $x \in K$ and $\alpha>0$.

Definition 2.3. A cone $K$ is said to be a global cone of instability for (2.1) if $x\left(x_{0}, t\right) \in K$ for arbitrary $x_{0} \in K$ and $t \geq 0$ and $\lim _{t \rightarrow \infty}\left\|x\left(x_{0}, t\right)\right\|=\infty$.

Definition 2.4. The zero solution $x \equiv 0$ of (2.1) is said to be globally unstable in a cone $K$ if $K$ is a global cone of instability for (2.1).

Now, we prove results analogous to the classical Chetaev theorem (see, e.g., [2]) on instability in a form suitable for our analysis. As usual, if $\mathcal{S}$ is a set, then $\partial \mathcal{S}$ denotes its boundary and $\overline{\mathcal{S}}$ its closure, that is, $\overline{\mathcal{S}}:=\mathcal{S} \cup \partial \mathcal{S}$.

Theorem 2.5. Let $V: \mathbb{R}^{n} \rightarrow \mathbb{R}, V(0, \ldots, 0)=0$ be a continuously differentiable function. Assume that the set

$$
K=\left\{x \in R^{n}: V(x)>0\right\}
$$

is a cone. If the full derivative of $V$ along the trajectories of (2.1) is positive for every $x \in K$, that is, if

$$
\dot{V}(x):=\operatorname{grad}^{T} V(x) f(x)>0, \quad x \in K,
$$

then $K$ is a global cone of instability for the system (2.1). 
Proof. Let $\varepsilon$ be a positive number. We define a neighborhood of the origin

$$
U_{\varepsilon}:=\left\{x \in R^{n}:\|x\|<\varepsilon\right\}
$$

and a constant

$$
M_{\varepsilon}:=\max _{x \in \bar{U}_{\varepsilon} \cap \bar{K}} V(x)
$$

Moreover, define a set

$$
W_{\delta}:=\left\{x \in \bar{U}_{\varepsilon} \cap \bar{K}, V(x) \geq \delta\right\}
$$

where $\delta$ is a positive number such that $\delta<M_{\varepsilon}$. Then, $W_{\delta} \neq \emptyset$.

Let $x_{0} \in W_{\delta} \cap K$, then $V\left(x_{0}\right)=\delta_{1} \in\left[\delta, M_{\varepsilon}\right]$. We show that there exists a $t=t_{T}=t_{T}\left(\varepsilon, x_{0}\right)$ such that $x\left(x_{0}, t_{T}\right) \notin \bar{U}_{\varepsilon}$ and $x\left(x_{0}, t_{T}\right) \in K$.

Suppose to the contrary that this is not true and $x\left(x_{0}, t\right) \in \bar{U}_{\varepsilon}$ for all $t \geq 0$. Since $\dot{V}(x)>0$, the function $V$ is increasing along the solutions of $(2.1)$. Thus $x\left(x_{0}, t\right)$ remains in $K$. Due to the compactness of $W_{\delta}$, there exists a positive value $\beta$ such that for $x\left(x_{0}, t\right) \in W_{\delta}$

$$
\frac{d}{d t} V\left(x\left(x_{0}, t\right)\right)=\operatorname{grad}^{T} V\left(x\left(x_{0}, t\right)\right) f\left(x\left(x_{0}, t\right)\right)>\beta .
$$

Integrating this inequality over the interval $[0, t]$, we get

$$
V\left(x\left(x_{0}, t\right)\right)-V\left(x_{0}\right)=V\left(x\left(x_{0}, t\right)\right)-\delta_{1}>\beta t .
$$

Then there exists a $t=t_{T}=t_{T}\left(\varepsilon, x_{0}\right)$ satisfying

$$
t_{T}>\frac{\left(M_{\varepsilon}-\delta_{1}\right)}{\beta}
$$

such that $V\left(x\left(x_{0}, t_{T}\right)\right)>M_{\varepsilon}$ and, consequently, $x\left(x_{0}, t_{T}\right) \notin \bar{U}_{\varepsilon}$. This is contrary to our supposition. Since $\varepsilon>0$ is arbitrary, we have

$$
\lim _{t \rightarrow \infty}\left\|x\left(x_{0}, t\right)\right\|=\infty
$$

that is, the zero solution is globally unstable, and $K$ is a global cone of instability.

Theorem 2.6. Let $V: \mathbb{R}^{n} \rightarrow \mathbb{R}$ be a continuously differentiable function and let $S, Z: \mathbb{R}^{n} \rightarrow \mathbb{R}$, $Z(0, \ldots, 0)=0$ be continuous functions such that $V=S \cdot Z$. Assume that the set

$$
K_{1}=\left\{x \in R^{n}: Z(x)>0\right\}
$$


is a cone, and $S(x)>0$ for any $x \in K_{1}$. If the full derivative (2.3) of $V$ along the trajectories of (2.1) is positive for every $x \in K_{1}$, that is, if $\dot{V}(x)>0$ for every $x \in K_{1}$, then $K_{1}$ is a global cone of instability for the system (2.1).

Proof. The proof is a modification of the proof of Theorem 2.5. Let $\varepsilon$ be a positive number. We define a neighborhood $U_{\varepsilon}$ of the origin by formula (2.4) and a constant

$$
M_{\varepsilon}:=\max _{x \in \bar{U}_{\varepsilon} \cap \overline{K_{1}}} V(x) .
$$

Moreover, define a set

$$
W_{\delta}:=\left\{x \in \bar{U}_{\varepsilon} \cap \overline{K_{1}}, V(x) \geq \delta\right\}
$$

where $\delta$ is a positive number such that $\delta<M_{\varepsilon}$. Then $W_{\delta} \neq \emptyset$.

Let $x_{0} \in W_{\delta} \cap K_{1}$. Then $V\left(x_{0}\right)=\delta_{1} \in\left[\delta, M_{\varepsilon}\right]$. We show that there exists a $t=t_{T}=$ $t_{T}\left(\varepsilon, x_{0}\right)$ such that $x\left(x_{0}, t_{T}\right) \notin \bar{U}_{\varepsilon}$ and $x\left(x_{0}, t_{T}\right) \in K_{1}$.

Suppose to the contrary that this is not true and $x\left(x_{0}, t\right) \in \bar{U}_{\varepsilon}$ for all $t \geq 0$. Since $\dot{V}(x)>0$, the function $V$ is increasing along the solutions of (2.1). Due to the compactness of $W_{\delta}$, there exists a positive value $\beta$ such that for $x\left(x_{0}, t\right) \in W_{\delta}$

$$
\frac{d}{d t} V\left(x\left(x_{0}, t\right)\right)=\operatorname{grad}^{T} V\left(x\left(x_{0}, t\right)\right) f\left(x\left(x_{0}, t\right)\right)>\beta .
$$

Integrating this inequality over interval $[0, t]$, we get

$$
V\left(x\left(x_{0}, t\right)\right)-V\left(x_{0}\right)=V\left(x\left(x_{0}, t\right)\right)-\delta_{1}=S\left(x\left(x_{0}, t\right)\right) Z\left(x\left(x_{0}, t\right)\right)-\delta_{1}>\beta t .
$$

Since $S\left(x\left(x_{0}, t\right)\right)>0$, the inequality

$$
Z\left(x\left(x_{0}, t\right)\right)>\frac{\delta_{1}+\beta t}{S\left(x\left(x_{0}, t\right)\right)}>0
$$

is an easy consequence of (2.15). Thus $x\left(x_{0}, t\right)$ remains in $K_{1}$. Apart from this, (2.15) also implies the existence of a $t=t_{T}=t_{T}\left(\varepsilon, x_{0}\right)$ satisfying

$$
t_{T}>\frac{\left(M_{\varepsilon}-\delta_{1}\right)}{\beta}
$$

such that $V\left(x\left(x_{0}, t_{T}\right)\right)>M_{\varepsilon}$. Consequently, $x\left(x_{0}, t_{T}\right) \notin \bar{U}_{\varepsilon}$. This is contrary to our supposition. Since $\varepsilon>0$ is arbitrary, we have

$$
\lim _{t \rightarrow \infty}\left\|x\left(x_{0}, t\right)\right\|=\infty
$$

that is, the zero solution is globally unstable and $K_{1}$ is a global cone of instability. 
Definition 2.7. A function $V$ satisfying all the properties indicated in Theorem 2.5 is called a Chetaev function for the system (2.1). A function $V$ satisfying all the properties indicated in Theorem 2.6 is called a Chetaev-type function for the system (2.1).

\subsection{Auxiliary Inequality}

Our results will be formulated in terms of global cones of instability. These will be derived using an auxiliary inequality valid in a given cone. Let $(x, y) \in \mathbb{R}^{2}$ and let $k$ be a positive number. We define a cone

$$
\mathcal{K}:=\left\{(x, y) \in \mathbb{R}^{2}: y>k|x|\right\}
$$

Lemma 2.8. Let $a, b, c, d$, and $k$ be given constants such that $b>0, d>0, k>0$, and $|c| \leq k d$. Assume, moreover, either

$$
|a| \leq k b
$$

or

$$
\begin{gathered}
|a|>k b, \\
|c| \neq k d, \quad k \geq \max \left\{\sqrt{\frac{|a+k b|}{c+k d}}, \sqrt{\frac{|a-k b|}{|c-k d|}}\right\},
\end{gathered}
$$

then

$$
a x^{3}+b x^{2} y+c x y^{2}+d y^{3}>0
$$

for every $(x, y) \in \mathcal{K}$.

Proof. We partition $\mathcal{K}$ into two disjoint cones

$$
\begin{aligned}
& \mathcal{K}_{1}:=\left\{(x, y) \in \mathbb{R}^{2}: y>k|x|, x>0\right\}, \\
& \mathcal{K}_{2}:=\left\{(x, y) \in \mathbb{R}^{2}: y>k|x|, x \leq 0\right\},
\end{aligned}
$$

and rewrite (2.23) as

$$
x\left(a x^{2}+c y^{2}\right)+y\left(b x^{2}+d y^{2}\right)>0
$$

We prove the validity of (2.23) in each of the two cones separately. 
The case of the cone $\mathcal{K}_{1}$. Suppose that (2.20) holds. Estimating the left-hand side of (2.25), we get

$$
\begin{aligned}
x\left(a x^{2}+c y^{2}\right)+y\left(b x^{2}+d y^{2}\right) & >x\left(a x^{2}+c y^{2}\right)+k x\left(b x^{2}+d y^{2}\right) \\
& =x\left[x^{2}(a+k b)+y^{2}(c+k d)\right]>0,
\end{aligned}
$$

and (2.23) holds.

If inequalities (2.21) and (2.22) are valid, then, estimating the left-hand side of (2.25), we get

$$
\begin{aligned}
x\left(a x^{2}+c y^{2}\right)+y\left(b x^{2}+d y^{2}\right) & >x\left(a x^{2}+c y^{2}\right)+k x\left(b x^{2}+d y^{2}\right) \\
& =x\left[x^{2}(a+k b)+y^{2}(c+k d)\right] \\
& \geq x\left[-|a+k b| x^{2}+(c+k d) y^{2}\right] \\
& =(c+k d) x\left[y^{2}-\frac{|a+k b|}{c+k d} x^{2}\right] \\
& =(c+k d) x\left[y-\sqrt{\frac{|a+k b|}{c+k d}} x\right]\left[y+\sqrt{\frac{|a+k b|}{c+k d}} x\right] \\
& =(c+k d) x^{2}\left[k-\sqrt{\frac{|a+k b|}{c+k d}}\right]\left[k+\sqrt{\frac{|a+k b|}{c+k d}}\right]
\end{aligned}
$$

$\geq 0$,

and (2.23) holds again.

The case of the cone $\mathcal{K}_{2}$. Suppose that (2.20) hold, then, estimating the left-hand side of (2.25), we get

$$
\begin{aligned}
x\left(a x^{2}+c y^{2}\right)+y\left(b x^{2}+d y^{2}\right) & =-|x|\left(a x^{2}+c y^{2}\right)+y\left(b x^{2}+d y^{2}\right) \\
& >-|x|\left(a x^{2}+c y^{2}\right)+k|x|\left(b x^{2}+d y^{2}\right) \\
& =-|x|\left[(a-k b) x^{2}+(c-k d) y^{2}\right] \\
& \geq 0,
\end{aligned}
$$

and (2.23) holds. 
If inequalities (2.21) and (2.22) are valid, then the estimation of (2.25) implies (we use

$$
\begin{aligned}
& x\left(a x^{2}+c y^{2}\right)+y\left(b x^{2}+d y^{2}\right) \\
& >-|x|\left[(a-k b) x^{2}+(c-k d) y^{2}\right] \\
& =|c-k d||x|\left[y^{2}-\frac{a-k b}{|c-k d|} x^{2}\right] \\
& =\left\{\begin{array}{l}
\geq 0 \\
|c-k d||x|\left[y-\sqrt{\frac{a-k b}{|c-k d|}} x\right]\left[y+\sqrt{\frac{a-k b}{|c-k d|}} x\right] \\
\geq|c-k d| x^{2}\left[k+\sqrt{\frac{a-k b}{|c-k d|}}\right]\left[k-\sqrt{\frac{a-k b}{|c-k d|}}\right] \geq 0 \quad \text { if } a-k b<0,
\end{array}\right.
\end{aligned}
$$

Hence, (2.23) holds again.

\section{Global Cone of Instability}

In this part we derive a result on the instability of system (1.5) in a cone. In order to properly formulate the results, we have to define some auxiliary vectors and matrices (some definitions copy the previous ones used in Introduction, but with a dimension of $n-1$ rather than $n$ ). We denote

$$
\begin{gathered}
x_{(n-1)}=\left(x_{1}, x_{2}, \ldots, x_{n-1}\right)^{T}, \\
b_{i}=\left(b_{1 n}^{i}, b_{2 n}^{i}, \ldots, b_{n-1, n}^{i}\right)^{T}, \quad i=1,2, \ldots, n, \\
\tilde{b}=\left(b_{n n}^{1}, b_{n n}^{2}, \ldots, b_{n n}^{n-1}\right)^{T} .
\end{gathered}
$$

Apart from this, we define symmetric $(n-1) \times(n-1)$ matrices

$$
B_{i}^{0}=\left\{b_{s q}^{i}\right\}, \quad i=1,2, \ldots, n, \quad s, q=1,2, \ldots, n-1,
$$


that is,

$$
\begin{aligned}
B_{i}^{0} & =\left(\begin{array}{cccc}
b_{11}^{i} & b_{12}^{i} & \cdots & b_{1, n-1}^{i} \\
b_{21}^{i} & b_{22}^{i} & \cdots & b_{2, n-1}^{i} \\
\cdots & \cdots & \cdots & \cdots \\
b_{n-1,1}^{i} & b_{n-1,2}^{i} & \cdots & b_{n-1, n-1}^{i}
\end{array}\right), \\
\widetilde{B} & =\left(\begin{array}{cccc}
b_{1 n}^{1} & b_{2 n}^{1} & \cdots & b_{n-1, n}^{1} \\
b_{1 n}^{2} & b_{2 n}^{2} & \cdots & b_{n-1, n}^{2} \\
\cdots & \cdots & \cdots & \cdots \\
b_{1 n}^{n-1} & b_{2 n}^{n-1} & \cdots & b_{n-1, n}^{n-1}
\end{array}\right) .
\end{aligned}
$$

Finally, we define an $(n-1) \times(n-1)^{2}$ matrix

$$
\bar{B}^{T}=\left\{\bar{B}_{1}^{T}, \bar{B}_{2}^{T}, \ldots, \bar{B}_{n-1}^{T}\right\}
$$

where $(n-1) \times(n-1)$ matrices $\bar{B}_{i}^{T}, i=1,2, \ldots, n-1$ are defined as

$$
\bar{B}_{i}^{T}=\left(\begin{array}{cccc}
b_{i 1}^{1} & b_{i 2}^{1} & \cdots & b_{i, n-1}^{1} \\
b_{i 1}^{2} & b_{i 2}^{2} & \cdots & b_{i, n-1}^{2} \\
\cdots & \cdots & \cdots & \cdots \\
b_{i 1}^{n-1} & b_{i 2}^{n-1} & \cdots & b_{i, n-1}^{n-1}
\end{array}\right) .
$$

We consider a matrix equation

$$
A_{0}^{T} H+H A_{0}=-C,
$$

where $H$ and $C$ are $(n-1) \times(n-1)$ matrices. It is well-known (see, e.g., [14]) that, for a given positive definite symmetric matrix $C$, (3.6) can be solved for a positive definite symmetric matrix $H$ if and only if the matrix $A_{0}$ is asymptotically stable.

Theorem 3.1 (Main result). Assume that the matrix $A_{0}$ is asymptotically stable, $b_{n n}^{n}>0$ and $h$ is a positive number. Let $C$ be an $(n-1) \times(n-1)$ positive definite symmetric matrix and $H$ be a related $(n-1) \times(n-1)$ positive definite symmetric matrix solving equation (3.6). Assume that the matrix $\left(-H \widetilde{B}^{T}-\widetilde{B} H+h\left(B_{n}^{0}\right)^{T}\right)$ is positive definite,

$$
\left\|2 h b_{n}-H \tilde{b}\right\| \leq \sqrt{\lambda_{\min }(H) h} \cdot b_{n n^{\prime}}^{n}
$$


and, in addition, one of the following conditions is valid:

either

$$
\left\|H \bar{B}^{T}\right\| \leq \sqrt{\frac{\lambda_{\min }(H)}{h}} \cdot \lambda_{\min }\left(-H \widetilde{B}^{T}-\widetilde{B} H+h\left(B_{n}^{0}\right)^{T}\right)
$$

or

$$
\left\|H \bar{B}^{T}\right\|>\sqrt{\frac{\lambda_{\min }(H)}{h}} \cdot \lambda_{\min }\left(-H \widetilde{B}^{T}-\widetilde{B} H+h\left(B_{n}^{0}\right)^{T}\right)
$$

a strong inequality holds in (3.7), and

$$
\sqrt{\frac{\lambda_{\min }(H)}{h}} \geq \max \left\{\sqrt{\tau_{1}}, \sqrt{\tau_{2}}\right\}
$$

where

$$
\begin{aligned}
& \tau_{1}=\frac{\left\|H \bar{B}^{T}\right\|-\sqrt{\lambda_{\min }(H) / h} \cdot \lambda_{\min }\left(-H \widetilde{B}^{T}-\tilde{B} H+h\left(B_{n}^{0}\right)^{T}\right)}{-\left\|2 h b_{n}-H \tilde{b}\right\|+\sqrt{\lambda_{\min }(H) h} \cdot b_{n n}^{n}}, \\
& \tau_{2}=\frac{\left\|H \bar{B}^{T}\right\|+\sqrt{\lambda_{\min }(H) / h} \cdot \lambda_{\min }\left(-H \widetilde{B}^{T}-\tilde{B} H+h\left(B_{n}^{0}\right)^{T}\right)}{\left\|2 h b_{n}-H \tilde{b}\right\|+\sqrt{\lambda_{\min }(H) h} \cdot b_{n n}^{n}} .
\end{aligned}
$$

Then the set

$$
K:=\left\{\left(x_{(n-1)}^{T}, x_{n}\right): \sqrt{h} x_{n}>\sqrt{x_{(n-1)}^{T} H x_{(n-1)}}\right\}
$$

is a global cone of instability for the system (1.5).

Proof. First we make auxiliary computations. For the reader's convenience, we recall that, for two $(n-1) \times(n-1)$ matrices $\mathcal{A}, \mathcal{A}_{1}$, two $1 \times(n-1)$ vectors $\ell, \ell_{1}$, two $(n-1) \times 1$ vectors $\mathcal{C}, \mathcal{C}_{1}$ and two $1 \times 1$ "matrices" $m, m_{1}$, the multiplicative rule

$$
\left(\begin{array}{ll}
\mathcal{A} & \mathcal{C} \\
\ell & m
\end{array}\right)\left(\begin{array}{ll}
A_{1} & \mathcal{C}_{1} \\
\ell_{1} & m_{1}
\end{array}\right)=\left(\begin{array}{ll}
A A_{1}+\mathcal{C} \ell_{1} & A C_{1}+C m_{1} \\
\ell A_{1}+m \ell_{1} & \ell C_{1}+m m_{1}
\end{array}\right)
$$

holds. This rule can be modified easily for the case of arbitrary rectangular matrices under the condition that all the products are well defined. 
We will rewrite system (1.5) in an equivalent form, suitable for further investigation. With this in mind, we define an $(n-1)^{2} \times(n-1)$ matrix $X_{(n-1)}$ as

$$
X_{(n-1)}^{T}=\left(X_{1(n-1)}^{T}, X_{2(n-1)}^{T}, \ldots, X_{n-1(n-1)}^{T}\right)
$$

where all the elements of the $(n-1) \times(n-1)$ matrices $X_{i(n-1)}^{T}, i=1,2, \ldots, n-1$ are equal to zero except the $i$ th row, which equals $x_{(n-1)}^{T}$, that is,

$$
X_{i(n-1)}^{T}=\left(\begin{array}{cccc}
0 & 0 & \cdots & 0 \\
\cdots & \cdots & \cdots & \cdots \\
0 & 0 & \cdots & 0 \\
x_{1} & x_{2} & \cdots & x_{n-1} \\
0 & 0 & \cdots & 0 \\
\cdots & \cdots & \cdots & \cdots \\
0 & 0 & \cdots & 0
\end{array}\right) .
$$

Moreover, we define $1 \times(n-1)$ vectors $Y_{i}, i=1,2, \ldots, n-1$ with components equal to zero except the $i$ th element, which equals $x_{n}$, that is,

$$
Y_{i}=\left(0, \ldots, 0, x_{n}, 0, \ldots, 0\right)
$$

and $(n-1) \times(n-1)$ zero matrix $\Theta$.

It is easy to see that matrices $X^{T}$ and $B$ in (1.5) can be expressed as

$$
\begin{aligned}
& X^{T}=\left(\begin{array}{ccccccc}
X_{1(n-1)}^{T} & Y_{1}^{T} & \cdots & X_{n-1(n-1)}^{T} & Y_{n-1}^{T} & \Theta & \theta \\
\theta^{T} & 0 & \cdots & \theta^{T} & 0 & x_{(n-1)}^{T} & x_{n}
\end{array}\right), \\
& B=\left(\begin{array}{cc}
B_{1}^{0} & b_{1} \\
b_{1}^{T} & b_{n n}^{1} \\
\cdots & \cdots \\
B_{n}^{0} & b_{n} \\
b_{n}^{T} & b_{n n}^{n}
\end{array}\right)
\end{aligned}
$$


Now we are able to rewrite the system (1.5) under the above assumption regarding the representation of the matrix $A$ in the form (1.10) in an equivalent form

$$
\begin{aligned}
\left(\begin{array}{c}
\dot{x}_{(n-1)} \\
\dot{x}_{n}
\end{array}\right)= & \left(\begin{array}{cc}
A_{0} & \theta \\
\theta^{T} & \lambda
\end{array}\right)\left(\begin{array}{c}
x_{(n-1)} \\
x_{n}
\end{array}\right) \\
& +\left(\begin{array}{ccccccc}
X_{1(n-1)}^{T} & Y_{1}^{T} & \cdots & X_{n-1(n-1)}^{T} & Y_{n-1}^{T} & \Theta & \theta \\
\theta^{T} & 0 & \cdots & \theta^{T} & 0 & x_{(n-1)}^{T} & x_{n}
\end{array}\right) \\
& \times\left(\begin{array}{cc}
B_{1}^{0} & b_{1} \\
b_{1}^{T} & b_{n n}^{1} \\
\cdots & \cdots \\
B_{n}^{0} & b_{n} \\
b_{n}^{T} & b_{n n}^{n}
\end{array}\right)\left(\begin{array}{c}
x_{(n-1)} \\
x_{n}
\end{array}\right) .
\end{aligned}
$$

Finally, since the equalities

$$
\begin{aligned}
\sum_{j=1}^{n-1} X_{j(n-1)}^{T} B_{j}^{0} & =\bar{B}^{T} X_{(n-1),} \\
\sum_{j=1}^{n-1} Y_{j}^{T} b_{j}^{T} & =\widetilde{B} x_{n,} \\
\sum_{j=1}^{n-1} X_{j(n-1)}^{T} b_{j} & =\widetilde{B} x_{(n-1)}, \\
\sum_{j=1}^{n-1} Y_{j}^{T} b_{n n}^{j} & =\tilde{b} x_{n}
\end{aligned}
$$

can be verified easily using (3.13), we have

$$
\left(\begin{array}{c}
\dot{x}_{(n-1)} \\
\dot{x}_{n}
\end{array}\right)=\left(\begin{array}{cc}
A_{0}+r_{11}\left(x_{(n-1)}^{T}, x_{n}\right) & r_{12}\left(x_{(n-1)}^{T}, x_{n}\right) \\
r_{21}\left(x_{(n-1)}^{T}, x_{n}\right) & \lambda+r_{22}\left(x_{(n-1)}^{T}, x_{n}\right)
\end{array}\right)\left(\begin{array}{c}
x_{(n-1)} \\
x_{n}
\end{array}\right)
$$


where

$$
\begin{gathered}
r_{11}\left(x_{(n-1)}^{T}, x_{n}\right)=\sum_{j=1}^{n-1}\left[X_{j(n-1)}^{T} B_{j}^{0}+Y_{j}^{T} b_{j}^{T}\right]=\bar{B}^{T} X_{(n-1)}+\widetilde{B} x_{n} \\
r_{12}\left(x_{(n-1)}^{T}, x_{n}\right)=\sum_{j=1}^{n-1}\left[X_{j(n-1)}^{T} b_{j}+Y_{j}^{T} b_{n n}^{j}\right]=\widetilde{B} x_{(n-1)}+\tilde{b} x_{n} \\
r_{21}\left(x_{(n-1)}^{T}, x_{n}\right)=x_{(n-1)}^{T} B_{n}^{0}+x_{n} b_{n}^{T} \\
r_{22}\left(x_{(n-1)}^{T}, x_{n}\right)=x_{(n-1)}^{T} b_{n}+x_{n} b_{n n}^{n} .
\end{gathered}
$$

The remaining part of the proof is based on Theorem 2.6 with a Chetaev-type function $V=$ $S \cdot Z$ and with suitable functions $S$ and $Z$. Such functions we define as

$$
V\left(x_{(n-1)}^{T}, x_{n}\right)=\left(\begin{array}{ll}
x_{(n-1)}^{T} & x_{n}
\end{array}\right)\left(\begin{array}{cc}
-H & \theta \\
\theta^{T} & h
\end{array}\right)\left(\begin{array}{c}
x_{(n-1)} \\
x_{n}
\end{array}\right)
$$

that is,

$$
\begin{gathered}
V\left(x_{(n-1)}^{T}, x_{n}\right)=-x_{(n-1)}^{T} H x_{(n-1)}+h x_{n}^{2} \\
S\left(x_{(n-1)}^{T}, x_{n}\right)=\sqrt{x_{(n-1)}^{T} H x_{(n-1)}}+\sqrt{h} x_{n} \\
Z\left(x_{(n-1)}^{T}, x_{n}\right)=-\sqrt{x_{(n-1)}^{T} H x_{(n-1)}}+\sqrt{h} x_{n} .
\end{gathered}
$$

We will verify the necessary properties. Obviously, $V=S \cdot Z$, the set

$$
\begin{aligned}
K_{1}: & =\left\{\left(x_{(n-1)}^{T}, x_{n}\right) \in \mathbb{R}^{n}: Z\left(x_{(n-1)}, x_{n}\right)>0\right\} \\
& =\left\{\left(x_{(n-1)}^{T}, x_{n}\right) \in \mathbb{R}^{n}: \sqrt{h} x_{n}>\sqrt{x_{(n-1)}^{T} H x_{(n-1)}}\right\}
\end{aligned}
$$

is a cone and $S\left(x_{(n-1)}^{T}, x_{n}\right)>0$ for every $\left(x_{(n-1)}^{T}, x_{n}\right) \in K_{1}$. 
The full derivative of $V$ (in the form (3.22)) along the trajectories of the system (1.5) (we use its transformed form (3.20)) equals

$$
\begin{aligned}
\dot{V}\left(x_{(n-1)}^{T}, x_{n}\right)= & \left(\begin{array}{ll}
\dot{x}_{(n-1)}^{T} & \dot{x}_{n}
\end{array}\right)\left(\begin{array}{cc}
-H & \theta \\
\theta^{T} & h
\end{array}\right)\left(\begin{array}{c}
x_{(n-1)} \\
x_{n}
\end{array}\right)+\left(\begin{array}{ll}
x_{(n-1)}^{T} & x_{n}
\end{array}\right)\left(\begin{array}{cc}
-H & \theta \\
\theta^{T} & h
\end{array}\right)\left(\begin{array}{c}
\dot{x}_{(n-1)} \\
\dot{x}_{n}
\end{array}\right) \\
= & \left(\begin{array}{ll}
x_{(n-1)}^{T} & x_{n}
\end{array}\right)\left(\begin{array}{cc}
A_{0}^{T}+r_{11}^{T}\left(x_{(n-1)}^{T}, x_{n}\right) & r_{21}^{T}\left(x_{(n-1)}^{T}, x_{n}\right) \\
r_{12}^{T}\left(x_{(n-1)}^{T}, x_{n}\right) & \lambda+r_{22}\left(x_{(n-1)}^{T}, x_{n}\right)
\end{array}\right)\left(\begin{array}{cc}
-H & \theta \\
\theta^{T} & h
\end{array}\right)\left(\begin{array}{c}
x_{(n-1)} \\
x_{n}
\end{array}\right) \\
& +\left(\begin{array}{cc}
x_{(n-1)}^{T} & x_{n}
\end{array}\right)\left(\begin{array}{cc}
-H & \theta \\
\theta^{T} & h
\end{array}\right)\left(\begin{array}{cc}
A_{0}+r_{11}\left(x_{(n-1)}^{T}, x_{n}\right) & r_{12}\left(x_{(n-1)}^{T}, x_{n}\right) \\
r_{21}\left(x_{(n-1)}^{T}, x_{n}\right) & \lambda+r_{22}\left(x_{(n-1)}^{T}, x_{n}\right)
\end{array}\right) \\
& \times\left(\begin{array}{c}
x_{(n-1)} \\
x_{n}
\end{array}\right) .
\end{aligned}
$$

Using formula (3.13), we get

$$
\dot{V}\left(x_{(n-1)}^{T}, x_{n}\right)=\left(\begin{array}{ll}
x_{(n-1)}^{T} & x_{n}
\end{array}\right)\left(\begin{array}{cc}
c_{11}\left(x_{(n-1)}^{T}, x_{n}\right) & c_{12}\left(x_{(n-1)}^{T}, x_{n}\right) \\
c_{21}\left(x_{(n-1)}^{T}, x_{n}\right) & c_{22}\left(x_{(n-1)}^{T}, x_{n}\right)
\end{array}\right)\left(\begin{array}{c}
x_{(n-1)} \\
x_{n}
\end{array}\right),
$$

where

$$
\begin{gathered}
c_{11}\left(x_{(n-1)}^{T}, x_{n}\right)=-\left[A_{0}+r_{11}\left(x_{(n-1)}^{T}, x_{n}\right)\right]^{T} H-H\left[A_{0}+r_{11}\left(x_{(n-1)}^{T}, x_{n}\right)\right], \\
c_{12}\left(x_{(n-1)}^{T}, x_{n}\right)=h r_{21}^{T}\left(x_{(n-1)}^{T}, x_{n}\right)-H r_{12}\left(x_{(n-1)}^{T}, x_{n}\right), \\
c_{21}\left(x_{(n-1)}^{T}, x_{n}\right)=h r_{21}\left(x_{(n-1)}^{T}, x_{n}\right)-r_{12}^{T}\left(x_{(n-1)}^{T}, x_{n}\right) H=c_{12}^{T}\left(x_{(n-1)}^{T}, x_{n}\right), \\
c_{22}\left(x_{(n-1)}^{T}, x_{n}\right)=2 h\left[\lambda+r_{22}\left(x_{(n-1)}^{T}, x_{n}\right)\right] .
\end{gathered}
$$

We reduce these formulas using (3.21). Then,

$$
\begin{gathered}
c_{11}\left(x_{(n-1)}^{T}, x_{n}\right)=-\left(A_{0}^{T} H+H A_{0}\right)-\left(\bar{B}^{T} X_{(n-1)}+\widetilde{B} x_{n}\right)^{T} H-H\left(\bar{B}^{T} X_{(n-1)}+\widetilde{B} x_{n}\right), \\
c_{12}\left(x_{(n-1)}^{T}, x_{n}\right)=h\left(x_{(n-1)}^{T} B_{n}^{0}+x_{n} b_{n}^{T}\right)^{T}-H\left(\widetilde{B} x_{(n-1)}+\tilde{b} x_{n}\right) \\
c_{21}\left(x_{(n-1)}^{T}, x_{n}\right)=h\left(x_{(n-1)}^{T} B_{n}^{0}+x_{n} b_{n}^{T}\right)-\left(\widetilde{B} x_{(n-1)}+\tilde{b} x_{n}\right)^{T} H \\
c_{22}\left(x_{(n-1)}^{T}, x_{n}\right)=2 h\left(\lambda+x_{(n-1)}^{T} b_{n}+x_{n} b_{n n}^{n}\right) .
\end{gathered}
$$


16

Abstract and Applied Analysis

The derivative (3.26) turns into

$$
\begin{aligned}
\dot{V}\left(x_{(n-1)}^{T}, x_{n}\right)= & x_{(n-1)}^{T} c_{11}\left(x_{(n-1)}^{T}, x_{n}\right) x_{(n-1)}+x_{(n-1)}^{T} c_{12}\left(x_{(n-1)}^{T}, x_{n}\right) x_{n} \\
& +x_{n} c_{21}\left(x_{(n-1)}^{T}, x_{n}\right) x_{(n-1)}+x_{n} c_{22}\left(x_{(n-1)}^{T}, x_{n}\right) x_{n} \\
= & x_{(n-1)}^{T}\left[-\left(A_{0}^{T} H+H A_{0}\right)-\left(\bar{B}^{T} X_{(n-1)}+\widetilde{B} x_{n}\right)^{T} H-H\left(\bar{B}^{T} X_{(n-1)}+\widetilde{B} x_{n}\right)\right] x_{(n-1)} \\
& +x_{(n-1)}^{T}\left[h\left(x_{(n-1)}^{T} B_{n}^{0}+x_{n} b_{n}^{T}\right)^{T}-H\left(\widetilde{B} x_{(n-1)}+\tilde{b} x_{n}\right)\right] x_{n} \\
& +x_{n}\left[h\left(x_{(n-1)}^{T} B_{n}^{0}+x_{n} b_{n}^{T}\right)-\left(\widetilde{B} x_{(n-1)}+\tilde{b} x_{n}\right)^{T} H\right] x_{(n-1)} \\
& +x_{n}\left[2 h\left(\lambda+x_{(n-1)}^{T} b_{n}+x_{n} b_{n n}^{n}\right)\right] x_{n} \\
= & -x_{(n-1)}^{T}\left(A_{0}^{T} H+H A_{0}\right) x_{(n-1)}+2 h \lambda x_{n}^{2} \\
& +2 h\left(x_{(n-1)^{T}}^{T} b_{n}+x_{n} b_{n n}^{n}\right) x_{n}^{2} . \\
& +2 x_{(n-1)}^{T}\left(h b_{n}-H \tilde{b}\right) x_{n}^{2}\left(\left(\bar{B}^{T} X_{(n-1)}\right)^{T} H+H \bar{B}^{T} X_{(n-1)}\right) x_{(n-1)} \\
& +x_{(n-1)}^{T}\left(2 h\left(B_{n}^{0}\right)^{T}-H \widetilde{B}-\widetilde{B} H\right) x_{(n-1)} x_{n} \\
& \left(\left(\widetilde{B} x_{n}\right)^{T} H+H \widetilde{B} x_{n}\right) x_{(n-1)} \\
& \\
& \\
& \\
& \\
&
\end{aligned}
$$

Finally, using (3.6), we get

$$
\begin{aligned}
\dot{V}\left(x_{(n-1)}^{T}, x_{n}\right)= & x_{(n-1)}^{T} C x_{(n-1)}+2 h \lambda x_{n}^{2}-2 x_{(n-1)}^{T} H \bar{B}^{T} X_{(n-1)} x_{(n-1)} \\
& +2 x_{(n-1)}^{T}\left[-H \widetilde{B}^{T}-\widetilde{B} H+h\left(B_{n}^{0}\right)^{T}\right] x_{(n-1)} x_{n}+2 x_{(n-1)}^{T}\left(2 h b_{n}-H \tilde{b}\right) x_{n}^{2}+2 h b_{n n}^{n} x_{n}^{3} .
\end{aligned}
$$


Let us find the conditions for the positivity of $\dot{V}\left(x_{(n-1)}^{T}, x_{n}\right)$ in the cone $K_{1}$. We use (3.30). If $\left(x_{(n-1)}^{T}, x_{n}\right) \in K_{1}$, then $x_{n} \geq 0$ and

$$
\begin{aligned}
\dot{V}\left(x_{(n-1)}^{T}, x_{n}\right) \geq & x_{(n-1)}^{T} C x_{(n-1)}+2 h \lambda x_{n}^{2}-2\left\|H \bar{B}^{T}\right\| \cdot\left\|x_{(n-1)}\right\|^{3} \\
& +2 \lambda_{\min }\left(-H \widetilde{B}^{T}-\widetilde{B} H+h\left(B_{n}^{0}\right)^{T}\right) \cdot\left\|x_{(n-1)}\right\|^{2} \cdot x_{n} \\
& -2\left\|2 h b_{n}-H \tilde{b}\right\| \cdot\left\|x_{(n-1)}\right\| \cdot x_{n}^{2}+2 h b_{n n}^{n} x_{n}^{3} .
\end{aligned}
$$

We set

$$
\begin{gathered}
a=-2\left\|H \bar{B}^{T}\right\|, \\
b=2 \lambda_{\min }\left(-H \tilde{B}^{T}-\tilde{B} H+h\left(B_{n}^{0}\right)^{T}\right), \\
c=-2\left\|2 h b_{n}-H \tilde{b}\right\|, \\
d=2 h b_{n n}^{n} .
\end{gathered}
$$

If

$$
a\left\|x_{(n-1)}\right\|^{3}+b\left\|x_{(n-1)}\right\|^{2} \cdot x_{n}+c\left\|x_{(n-1)}\right\| \cdot x_{n}^{2}+d x_{n}^{3}>0
$$

in $K_{1}$, then $\dot{V}\left(x_{(n-1)}^{T}, x_{n}\right)>0$ since $C$ is a positive definite matrix and

$$
x_{(n-1)}^{T} C x_{(n-1)}+2 h \lambda x_{n}^{2} \geq \lambda_{\min }(C)\left\|x_{(n-1)}\right\|^{2}+2 h \lambda x_{n}^{2}>0 .
$$

If $\left(x_{(n-1)}^{T}, x_{n}\right) \in K_{1}$, then

$$
\begin{gathered}
x_{n}>\sqrt{\frac{x_{(n-1)}^{T} H x_{(n-1)}}{h}} \geq \sqrt{\frac{\lambda_{\min }(H)}{h}} \cdot\left\|x_{(n-1)}\right\|, \\
K_{1} \subset \mathcal{K}^{*}:=\left\{\left(x_{(n-1)}^{T}, x_{n}\right) \in \mathbb{R}^{n}: x_{n}>\sqrt{\frac{\lambda_{\min }(H)}{h}} \cdot\left\|x_{(n-1)}\right\|\right\} .
\end{gathered}
$$

Now, we use Lemma 2.8 with $\mathcal{K}=\mathcal{K}^{*}, y=x_{n}, x=\left\|x_{(n-1)}\right\|$, with coefficients $a, b, c$, and $d$ defined by formula (3.32) and with $k:=\sqrt{\lambda_{\min }(H) / h}$.

Obviously $|c| \leq k d$ because, due to (3.7), inequality

$$
\left\|2 h b_{n}-H \tilde{b}\right\| \leq \sqrt{\lambda_{\min }(H) h} \cdot b_{n n}^{n}
$$


holds. Moreover, $|a| \leq k b$ if (3.8) holds, that is, if

$$
\left\|H \bar{B}^{T}\right\| \leq \sqrt{\frac{\lambda_{\min }(H)}{h}} \cdot \lambda_{\min }\left(-H \widetilde{B}^{T}-\tilde{B} H+h\left(B_{n}^{0}\right)^{T}\right) .
$$

Further, $|a|>k b$ if (3.9) holds, that is, if

$$
\left\|H \bar{B}^{T}\right\|>\sqrt{\frac{\lambda_{\min }(H)}{h}} \cdot \lambda_{\min }\left(-H \tilde{B}^{T}-\tilde{B} H+h_{n}\left(B_{n}^{0}\right)^{T}\right),
$$

and (2.22) holds due to (4.10) and the condition $|c| \neq k d$. Thus the assumptions of Lemma 2.8 are true, the inequality (3.33) holds in the cone $\mathcal{K}^{*}$ and, due to embedding (3.36), in the cone $K_{1}$ as well.

All the assumptions of Theorem 2.6 are fulfilled with regard to system (1.5) and the theorem is proved, because $K_{1}=K$.

Remark 3.2. We will focus our attention to Lemma 2.8 about the positivity of a third-degree polynomial in two variables in the cone $\mathcal{K}$. We used it to estimate the derivative $\dot{V}$ expressed by formula (3.30). Obviously, there are other possibilities of estimating its sign. Let us demonstrate one of them. Let us, for example, estimate the right-hand side of (3.31) in the cone $K_{1}$ using inequality (3.35), then

$$
\begin{aligned}
\dot{V}\left(x_{(n-1)}^{T}, x_{n}\right) \geq & x_{(n-1)}^{T} C x_{(n-1)}+2 h \lambda x_{n}^{2}-2\left\|H \bar{B}^{T}\right\| \cdot\left\|x_{(n-1)}\right\|^{3} \\
& +2 \lambda_{\min }\left(-H \widetilde{B}^{T}-\widetilde{B} H+h\left(B_{n}^{0}\right)^{T}\right) \cdot\left\|x_{(n-1)}\right\|^{2} \cdot x_{n} \\
& -2\left\|2 h b_{n}-H \tilde{b}\right\| \cdot\left\|x_{(n-1)}\right\| \cdot x_{n}^{2}+2 h b_{n n}^{n} x_{n}^{3} \\
\geq & \lambda_{\min }(C)\left\|x_{(n-1)}\right\|^{2}+2 h \lambda x_{n}^{2}-2\left\|H \bar{B}^{T}\right\| \cdot\left\|x_{(n-1)}\right\|^{3} \\
& +2 \sqrt{\frac{\lambda_{\min }(H)}{h}} \cdot \lambda_{\min }\left(-H \widetilde{B}^{T}-\widetilde{B} H+h\left(B_{n}^{0}\right)^{T}\right) \cdot\left\|x_{(n-1)}\right\|^{3} \\
& -2\left\|2 h b_{n}-H \tilde{b}\right\| \cdot\left\|x_{(n-1)}\right\| \cdot x_{n}^{2}+2 \sqrt{\frac{\lambda_{\min }(H)}{h}} \cdot\left\|x_{(n-1)}\right\| \cdot h b_{n n}^{n} \cdot x_{n}^{2}
\end{aligned}
$$

and the positivity of $\dot{V}\left(x_{(n-1)}^{T}, x_{n}\right)$ will be guaranteed if

$$
\begin{gathered}
\left\|H \bar{B}^{T}\right\| \leq \sqrt{\frac{\lambda_{\min }(H)}{h}} \cdot \lambda_{\min }\left(-H \widetilde{B}^{T}-\widetilde{B} H+h\left(B_{n}^{0}\right)^{T}\right), \\
\left\|2 h b_{n}-H \tilde{b}\right\| \leq \sqrt{\lambda_{\min }(H) h} \cdot b_{n n}^{n} .
\end{gathered}
$$


We see that this approach produces only one set of inequalities for the positivity of $\dot{V}\left(x_{(n-1)}^{T}, x_{n}\right)$, namely the case when (3.7) and (3.8) holds. Unfortunately, using such approach, we are not able to detect the second case (3.7) and (3.9) when $\dot{V}\left(x_{(n-1)}^{T}, x_{n}\right)$ is positive. This demonstrates the advantage of detailed estimates using the above third-degree polynomial in two variables.

\section{Planar Case}

Now we consider a particular case of the system (1.5) for $n=2$. This means that, in accordance with (1.5) and (1.10), we consider a system

$$
\begin{aligned}
& \dot{x}_{1}(t)=a x_{1}(t)+b_{11}^{1} x_{1}^{2}(t)+2 b_{12}^{1} x_{1}(t) x_{2}(t)+b_{22}^{1} x_{2}^{2}(t) \\
& \dot{x}_{2}(t)=\lambda x_{2}(t)+b_{11}^{2} x_{1}^{2}(t)+2 b_{12}^{2} x_{1}(t) x_{2}(t)+b_{22}^{2} x_{2}^{2}(t)
\end{aligned}
$$

where $a<0$ and $\lambda>0$. The solution of matrix equation (3.6) for $A_{0}=(a), H=\left(h_{11}\right)$, and $C=(c)$ with $c>0$, that is,

$$
\left(a h_{11}\right)+\left(h_{11} a\right)=-(c)
$$

gives

$$
H=\left(h_{11}\right)=\left(-\frac{c}{2 a}\right)
$$

with $h_{11}=-c / 2 a>0$. The set $K$ defined by (3.12) where $h>0$ and $x_{(n-1)}=x_{1}$ reduces to

$$
K=\left\{\left(x_{1}, x_{2}\right): x_{2}>\sqrt{\frac{c}{2|a| h}} \cdot\left|x_{1}\right|\right\}
$$

Now, from Theorem 3.1, we will deduce sufficient conditions indicating $K$ being a global cone of instability for system (4.1). In our particular case, we have

$$
\begin{gathered}
b_{i}=\left(b_{12}^{i}\right), \quad i=1,2, \quad \tilde{b}=\left(b_{22}^{1}\right), \\
B_{i}^{0}=\left(b_{11}^{i}\right), \quad i=1,2, \quad \widetilde{B}=\left(b_{12}^{1}\right), \quad \bar{B}^{T}=\left(b_{11}^{1}\right)=B_{1}^{0} .
\end{gathered}
$$


Now, we compute all necessary expressions used in Theorem 3.1. We have

$$
\begin{aligned}
& -H \widetilde{B}^{T}-\widetilde{B} H+h\left(B_{n}^{0}\right)^{T}=-\left(-\frac{c}{2 a}\right)\left(b_{12}^{1}\right)-\left(b_{12}^{1}\right)\left(-\frac{c}{2 a}\right)+h\left(b_{11}^{2}\right)=\left(h b_{11}^{2}-\frac{c}{|a|} b_{12}^{1}\right), \\
& \left\|2 h b_{n}-H \tilde{b}\right\|=\left|2 h b_{12}^{2}-\frac{c}{2|a|} b_{22}^{1}\right|, \\
& \sqrt{\lambda_{\min }(H) h}=\sqrt{\frac{c h}{2|a|}} \\
& \sqrt{\frac{\lambda_{\min }(H)}{h}}=\sqrt{\frac{c}{2|a| h}} \\
& \left\|H \bar{B}^{T}\right\|=\left|\frac{c}{2|a|} b_{11}^{1}\right|=\frac{c}{2|a|}\left|b_{11}^{1}\right| \text {, } \\
& \lambda_{\min }\left(-H \tilde{B}^{T}-\tilde{B} H+h\left(B_{n}^{0}\right)^{T}\right)=h b_{11}^{2}-\frac{c}{|a|} b_{12}^{1}, \\
& \tau_{1}=\frac{\left\|H \bar{B}^{T}\right\|-\sqrt{\lambda_{\min }(H) / h} \cdot \lambda_{\min }\left(-H \widetilde{B}^{T}-\tilde{B} H+h\left(B_{n}^{0}\right)^{T}\right)}{-\left\|2 h b_{n}-H \tilde{b}\right\|+\sqrt{\lambda_{\min }(H) h} \cdot b_{n n}^{n}} \\
& =\frac{(c / 2|a|)\left|b_{11}^{1}\right|-\sqrt{c / 2|a| h} \cdot\left(h b_{11}^{2}-(c /|a|) b_{12}^{1}\right)}{-\left|2 h b_{12}^{2}-(c / 2|a|) b_{22}^{1}\right|+\sqrt{c h / 2|a|} \cdot b_{22}^{2}}, \\
& \tau_{2}=\frac{\left\|H \bar{B}^{T}\right\|+\sqrt{\lambda_{\min }(H) / h} \cdot \lambda_{\min }\left(-H \tilde{B}^{T}-\tilde{B} H+h\left(B_{n}^{0}\right)^{T}\right)}{\left\|2 h b_{n}-H \tilde{b}\right\|+\sqrt{\lambda_{\min }(H) h} \cdot b_{n n}^{n}} \\
& =\frac{(c / 2|a|)\left|b_{11}^{1}\right|+\sqrt{(c / 2|a| h)} \cdot\left(h b_{11}^{2}-(c /|a|) b_{12}^{1}\right)}{\left|2 h b_{12}^{2}-(c / 2|a|) b_{22}^{1}\right|+\sqrt{(c h / 2|a|)} \cdot b_{22}^{2}} \text {. }
\end{aligned}
$$

Theorem 4.1 (Planar Case). Assume that $a<0, b_{22}^{2}>0, h>0, c>0$ and $h b_{11}^{2}|a|>c b_{12}^{1}$. Let

$$
\left|2 h b_{12}^{2}-\frac{c}{2|a|} b_{22}^{1}\right| \leq \sqrt{\frac{c h}{2|a|}} \cdot b_{22}^{2},
$$

and, in addition, one of the following conditions is valid:

either

$$
\frac{c}{2|a|}\left|b_{11}^{1}\right| \leq \sqrt{\frac{c}{2|a| h}} \cdot\left(h b_{11}^{2}-\frac{c}{|a|} b_{12}^{1}\right)
$$


or

$$
\frac{c}{2|a|}\left|b_{11}^{1}\right|>\sqrt{\frac{c}{2|a| h}} \cdot\left(h b_{11}^{2}-\frac{c}{|a|} b_{12}^{1}\right)
$$

strong inequality holds in (4.7), and

$$
\sqrt{\frac{c}{2|a| h}} \geq \max \left\{\sqrt{\tau_{1}}, \sqrt{\tau_{2}}\right\}
$$

where $\tau_{1}$ and $\tau_{1}$ are defined by (4.6). Then the set $K$ defined by (4.4) is a global cone of instability for the system (4.1).

It is easy to see that the choice $h=1, c=|a|$ significantly simplifies all assumptions. Therefore we give such a particular case of Theorem 4.1.

Corollary 4.2 (Planar Case). Assume that $a<0, b_{22}^{2}>0$ and $b_{11}^{2}>b_{12}^{1}$. Let

$$
\left|2 b_{12}^{2}-\frac{1}{2} b_{22}^{1}\right| \leq \frac{1}{\sqrt{2}} \cdot b_{22}^{2}
$$

and, in addition, one of the following conditions is valid:

either

$$
\frac{1}{2}\left|b_{11}^{1}\right| \leq \frac{1}{\sqrt{2}} \cdot\left(b_{11}^{2}-b_{12}^{1}\right)
$$

or

$$
\frac{1}{2}\left|b_{11}^{1}\right|>\frac{1}{\sqrt{2}} \cdot\left(b_{11}^{2}-b_{12}^{1}\right),
$$

strong inequality holds in (4.11), and

$$
\frac{1}{\sqrt{2}} \geq \max \left\{\sqrt{\tau_{1}}, \sqrt{\tau_{2}}\right\}
$$

where

$$
\tau_{1}=\frac{(1 / 2)\left|b_{11}^{1}\right|-(1 / \sqrt{2}) \cdot\left(b_{11}^{2}-b_{12}^{1}\right)}{-\left|2 b_{12}^{2}-(1 / 2) b_{22}^{1}\right|+(1 / \sqrt{2}) \cdot b_{22}^{2}}, \quad \tau_{2}=\frac{(1 / 2)\left|b_{11}^{1}\right|+(1 / \sqrt{2}) \cdot\left(b_{11}^{2}-b_{12}^{1}\right)}{\left|2 b_{12}^{2}-(1 / 2) b_{22}^{1}\right|+(1 / \sqrt{2}) \cdot b_{22}^{2}}
$$


Then the set

$$
K=\left\{\left(x_{1}, x_{2}\right): x_{2}>\frac{1}{\sqrt{2}} \cdot\left|x_{1}\right|\right\}
$$

is a global cone of instability for the system (4.1).

Example 4.3. The set $K$ defined by (4.16) is a global cone of instability for the system

$$
\begin{gathered}
\dot{x}_{1}(t)=a x_{1}(t)+x_{1}^{2}(t)+2 \sqrt{2} x_{1}(t) x_{2}(t)+x_{2}^{2}(t), \\
\dot{x}_{2}(t)=\lambda x_{2}(t)+2 \sqrt{2} x_{1}^{2}(t)+2 x_{1}(t) x_{2}(t)+2 \sqrt{2} x_{2}^{2}(t),
\end{gathered}
$$

where $a<0$ and $\lambda>0$ since inequalities (4.11) and (4.12) in Corollary 4.2 hold.

Example 4.4. The set $K$ defined by (4.16) is a global cone of instability for the system

$$
\begin{gathered}
\dot{x}_{1}(t)=a x_{1}(t)+4 x_{1}^{2}(t)+2 \sqrt{2} x_{1}(t) x_{2}(t)+x_{2}^{2}(t), \\
\dot{x}_{2}(t)=\lambda x_{2}(t)+2 \sqrt{2} x_{1}^{2}(t)+2 x_{1}(t) x_{2}(t)+20 \sqrt{2} x_{2}^{2}(t),
\end{gathered}
$$

where $a<0$ and $\lambda>0$ since inequalities (4.11), (4.13), (4.14) in Corollary 4.2 hold.

\section{Acknowledgments}

This research was supported by Grants nos. P201/11/0768 and P201/10/1032 of Czech Grant Agency, and by the Council of Czech Government nos. MSM 0021630503, MSM 0021630519, and MSM 0021630529, and by Grant FEKT-S-11-2-921 of Faculty of Electrical Engineering and Communication.

\section{References}

[1] A. M. Lyapunov, General Problem of Stability of Motion, Gostekhizdat, Moscow, Russia, 1950.

[2] N. G. Chetaev, Dynamic Stability, Nauka, Moscow, Russia, 1965.

[3] B. P. Demidovič, Lectures on Mathematical Stability Theory, Nauka, Moscow, Russia, 1967.

[4] X. Liao, L. Wang, and P. Yu, Stability of Dynamical Systems, vol. 5 of Monograph Series on Nonlinear Science and Complexity, Elsevier, Amsterdam, The Netherlands, 2007.

[5] V. M. Matrosov and A. I. Malikov, "The development of the ideas of A. M. Lyapunov over one hundred years: 1892-1992," Izvestiya Vysshikh Uchebnykh Zavedeniǔ. Matematika, no. 4, pp. 3-47, 1993 (Russian).

[6] K. P. Persidskiy, "About dynamic stability in the first approximation," Matematicheskii Sbornik, vol. 40, pp. 284-293, 1933 (Russian).

[7] S. K. Persidskiy, "Investigation by the second Lyapunov method of the property of having fixed sign in a cone of homogeneous polynomials of the second and third power," Dynam. Sys., vol. 33, pp. 3-6, 1994.

[8] N. Rouche, P. Habets, and M. Laloy, Stability Theory by Liapunov's Method, vol. 2 of Applied Mathematical Sciences, Springer, New York, NY, USA, 1977.

[9] V. V. Rumyantsev, "Development of research in the USSR in the theory of stability of motion," Differential Equations, vol. 19, no. 5, pp. 739-776, 1983 (Russian). 
[10] V. E. Slyusarchuk, Instability of Solutions of Evolutionary Equations, NUVGP, Rovno, Ukraine, 2004.

[11] F. P. Gantmacher, The Theory of Matrices, vol. 1, AMS Chelsea, Providence, RI, USA, 2002.

[12] D. I. Martynjuk, Lectures on the Qualitative Theory of Difference Equations, Naukova Dumka, Kiev, Ukraine, 1972.

[13] J. Diblík and D. Khusainov, "Stability of an autonomous system with quadratic right-hand side in the critical case," Nonlinear Dynamics and Systems Theory, vol. 2, no. 2, pp. 145-156, 2002.

[14] T. A. Burton, Stability and Periodic Solutions of Ordinary and Functional Differential Equations, Dover, Mineola, NY, USA, 2005. 


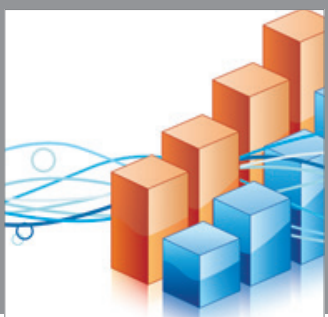

Advances in

Operations Research

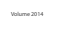

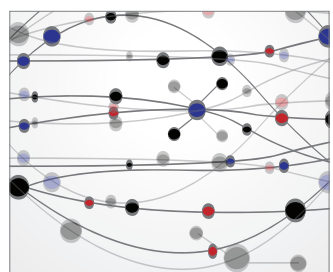

\section{The Scientific} World Journal
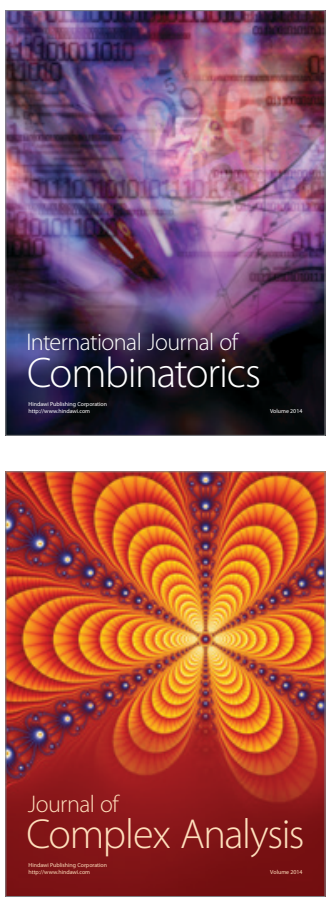

International Journal of

Mathematics and

Mathematical

Sciences
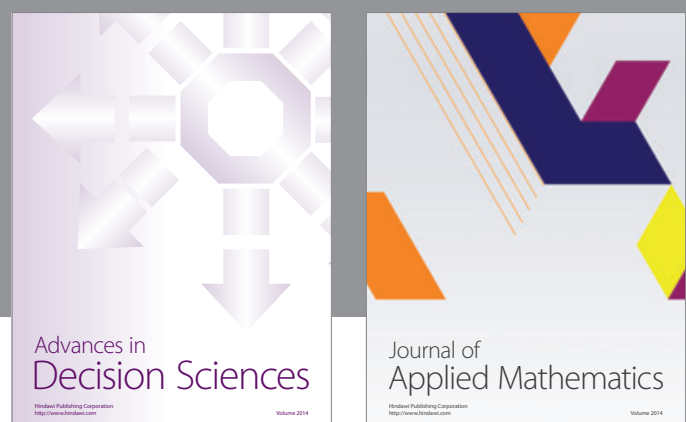

Journal of

Applied Mathematics
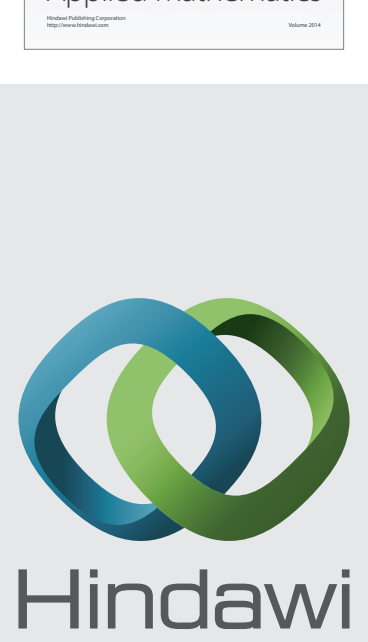

Submit your manuscripts at http://www.hindawi.com
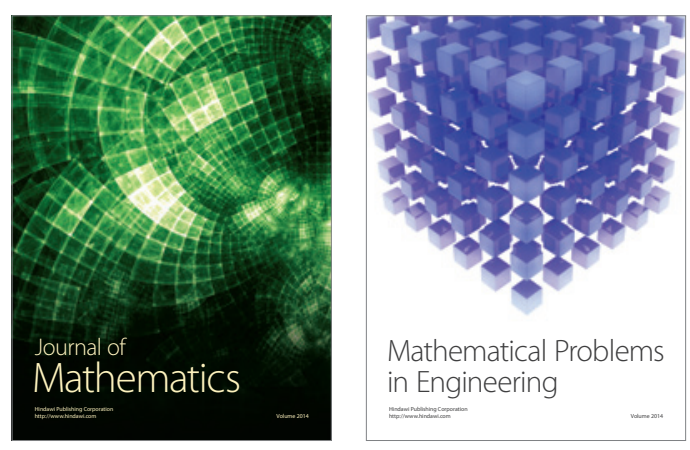

Mathematical Problems in Engineering
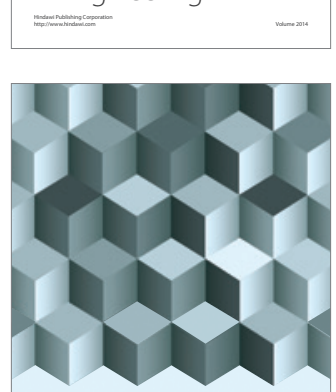

Journal of

Function Spaces
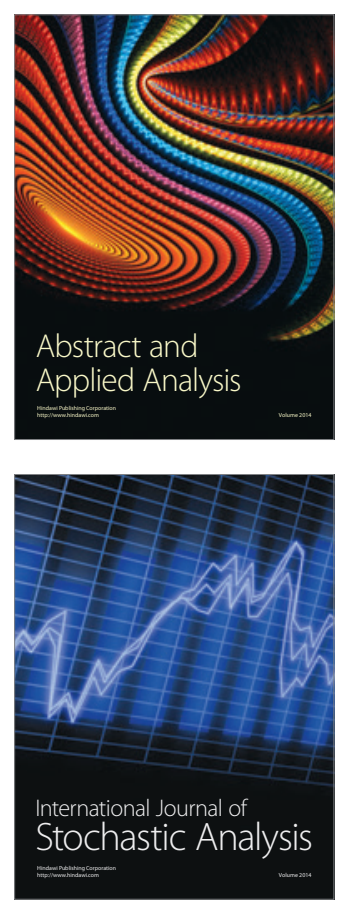

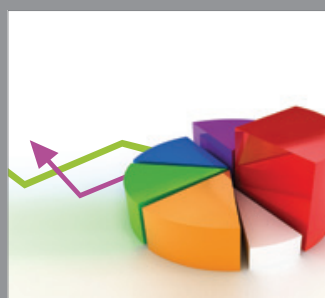

ournal of

Probability and Statistics

Promensencen
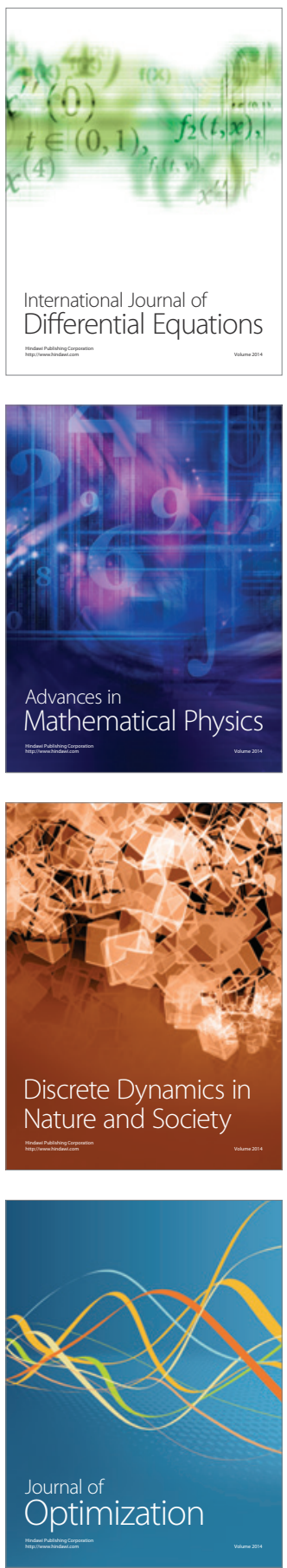